Proyecciones Journal of Mathematics

Vol. 31, No 4, pp. 345-354, December 2012.

Universidad Católica del Norte

Antofagasta - Chile

\title{
Matrix representation of the q-Jacobsthal numbers
}

\author{
Gamaliel Cerda-Morales \\ P. Universidad Católica de Valparaśo, Chile \\ Received : September 2012. Accepted : October 2012
}

\begin{abstract}
In this paper, we consider a q-Jacobsthal sequence $\left\{J_{q, n}\right\}$, with initial conditions $J_{q, 0}=0$ and $J_{q, 1}=1$. Then give a generating matrix for the terms of sequence $\left\{J_{q, k n}\right\}$ for a positive integer $k$. With the aid of this matrix, we derive some new identities for the sequence $\left\{J_{q, k n}\right\}$.
\end{abstract}

Subjclass : 11B39, 11B37, 15A36.

Keywords : q-Jacobsthal numbers, q-Jacobsthal-Lucas numbers, matrix methods. 


\section{Introduction}

In [H1], Horadam introduce a sequence $\left\{W_{n}(a, b ; p, q)\right\}$, or briefly $\left\{W_{n}\right\}$, defined by the recurrence relation

$$
W_{n}=p W_{n-1}-q W_{n-2}, n \geq 2,
$$

with $W_{0}=a, W_{1}=b$, where $a, b, p$ and $q$ are integers with $p>0, q \neq 0$.

We are interested in the following two special cases of $\left\{W_{n}\right\}$, the $q$ Jacohsthal sequence $\left\{J_{q, n}\right\}$ defined by

$$
J_{q, n}=J_{q, n-1}-q J_{q, n-2}, J_{q, 0}=0, J_{q, 1}=1, n \geq 2,
$$

and the $q$-Jacobsthal-Lucas sequence $\left\{j_{q, n}\right\}$ defined by

$$
j_{q, n}=j_{q, n-1}-q j_{q, n-2}, j_{q, 0}=2, j_{q, 1}=1, n \geq 2 .
$$

The above recurrences involve the characteristic equation

$$
x^{2}-x+q=0
$$

with roots $\alpha_{q}=\frac{1+\sqrt{1-4 q}}{2}$ and $\beta_{q}=\frac{1+\sqrt{1-4 q}}{2}$. Explicit closed form expressions for $J_{q, n}$ and $j_{q, n}$ are $(n \geq 1)$

$$
J_{q, n}=\frac{\alpha_{q}^{n}-\beta_{q}^{n}}{\alpha_{q}-\beta_{q}}
$$

and

$$
j_{q, n}=\alpha_{q}^{n}+\beta_{q}^{n} .
$$

Particular cases of the previous definition are:

- If $q=-1$, the classic Fibonacci sequence appears by $F_{0}=0, F_{1}=1$ and $F_{n+1}=F_{n}+F_{n-1}$ for $n \geq 1:\left\{F_{n}\right\}_{n \in N}=\{0,1,1,2,3,5,8, \ldots\}$.

- If $q=-2$, the Jacobsthal sequence introduced in [H2], and defined by $J_{n+1}=J_{n}+2 J_{n-1}$ for $n \geq 1, J_{0}=0, J_{1}=1:\left\{J_{n}\right\}_{n \in N}=$ $\{0,1,1,3,5,11, \ldots\}$.

We define $J_{q}$ be the $2 \times 2$ matrix

$$
J_{q}=\left[\begin{array}{rr}
1 & -q \\
1 & 0
\end{array}\right],
$$


then for an integer $n$ with $n \geq 1, J_{q}^{n}$ has the form

$$
J_{q}^{n}=\left[\begin{array}{lr}
J_{q, n+1} & -q J_{q, n} \\
J_{q, n} & -q J_{q, n-1}
\end{array}\right] .
$$

The particular case $q=-2$, was introduced by Köken and Bozkurt in [KB2, KB3]. Moreover, they have obtained the Cassini formula for the Jacobsthal numbers. In this paper, we study new relations on $q$-Jacobsthal sequence, using the matrix $J_{q}$ defined in (1.7).

Initially, the $q$-Jacobsthal numbers are defined for $n \geq 0$ but their existence for $n<0$ is readily extended, yielding

$$
J_{q,-n}=-q^{-n} J_{q, n} \text { and } j_{q,-n}=q^{-n} j_{q, n} .
$$

For $n \geq 2$ and a fixed positive integer $k$, in [KS] the authors study the sequence $\left\{W_{q, k n}(x)\right\}$ and prove the following relation:

$$
\begin{aligned}
& J_{q, k n}=j_{q, k} J_{q, k(n-1)}-q^{k} J_{q, k(n-2)}, \\
& j_{q, k n}=j_{q, k} j_{q, k(n-1)}-q^{k} j_{q, k(n-2)},
\end{aligned}
$$

where the initial conditions of the sequences $\left\{J_{q, n}\right\}$ and $\left\{j_{q, n}\right\}$ are 0 and $\left\{J_{q, k}\right\}$, and 2 and $\left\{j_{q, k}\right\}$, respectively.

If $\alpha_{q, k}$ and $\beta_{q, k}$ are the roots of equation $\lambda^{2}-j_{q, k} \lambda+q^{k}=0$, then the Binet formulas of the sequences $\left\{J_{q, k n}\right\}$ and $\left\{j_{q, k n}\right\}$ are given by

$$
J_{q, k n}=J_{q, k}\left(\frac{\alpha_{q, k}^{n}-\beta_{q, k}^{n}}{\alpha_{q, k}-\beta_{q, k}}\right) \text { and } j_{q, k n}=\alpha_{q, k}^{n}+\beta_{q, k}^{n},
$$

respectively. It is clear that $\alpha_{q, 1}=\alpha_{q}$ and $\beta_{q, 1}=\beta_{q}$.

From the Binet formulas, one can see that $J_{q, 2 k n}=j_{q, k n} J_{q, k n}$.

\section{Companion matrix for the sequence $\left\{J_{q, k n}\right\}$}

In this section, we define a $2 \times 2$ matrix $A_{q}$ and then we give some new results for the $q$-Jacobsthal numbers $J_{q, k n}$ by matrix methods.

Define the $2 \times 2$ matrix $A_{q}$ as follows:

$$
A_{q}=\left[\begin{array}{cr}
j_{q, k} & -q^{k} \\
1 & 0
\end{array}\right] .
$$

By an inductive argument and using (1.9), we get

Proposition 2.1. For any integer $n \geq 1$ holds:

$$
A_{q}^{n}=\frac{1}{J_{q, k}}\left[\begin{array}{lr}
J_{q, k(n+1)} & -q^{k} J_{q, k n} \\
J_{q, k n} & -q^{k} J_{q, k(n-1)}
\end{array}\right] .
$$


Proof. (By induction). For $n=1$ :

$$
A_{q}^{1}=\left[\begin{array}{cr}
j_{q, k} & -q^{k} \\
1 & 0
\end{array}\right]=\frac{1}{J_{q, k}}\left[\begin{array}{ll}
J_{q, 2 k} & -q^{k} J_{q, k} \\
J_{q, k} & -q^{k} J_{q, 0}
\end{array}\right]
$$

since $J_{q, 0}=0$ and $J_{q, 2 k}=j_{q, k} J_{q, k}$. Let us suppose that the formula is true for $n-1$ :

$$
A_{q}^{n-1}=\frac{1}{J_{q, k}}\left[\begin{array}{ll}
J_{q, k n} & -q^{k} J_{q, k(n-1)} \\
J_{q, k(n-1)} & -q^{k} J_{q, k(n-2)}
\end{array}\right] .
$$

Then, $\mathrm{A}_{q}^{n}=A_{q}^{n-1} A_{q}^{1}=\frac{1}{J_{q, k}}\left[\begin{array}{ll}J_{q, k n} & -q^{k} J_{q, k(n-1)} \\ J_{q, k(n-1)} & -q^{k} J_{q, k(n-2)}\end{array}\right]\left[\begin{array}{rr}j_{q, k} & -q^{k} \\ 1 & 0\end{array}\right]$ $=\frac{1}{J_{q, k}}\left[\begin{array}{lr}j_{q, k} J_{q, k n}-q^{k} J_{q, k(n-1)} & -q^{k} J_{q, k n} \\ j_{q, k} J_{q, k(n-1)}-q^{k} J_{q, k(n-2)} & -q^{k} J_{q, k(n-1)}\end{array}\right]$ $=\frac{1}{J_{q, k}}\left[\begin{array}{lr}J_{q, k(n+1)} & -q^{k} J_{q, k n} \\ J_{q, k n} & -q^{k} J_{q, k(n-1)}\end{array}\right]$

Clearly the matrix $A_{q}^{n}$ satisfies the recurrence relation, for $n \geq 1$

$$
A_{q}^{n+1}=j_{q, k} A_{q}^{n}-q^{k} A_{q}^{n-1},
$$

where $A_{q}^{0}=I_{2}, A_{q}^{1}=A_{q}$ and $I_{2}$ is the $2 \times 2$ unit matrix.

In this study, we define the $q$-Jacobsthal-Lucas $j_{q}$-matrix by

$$
j_{q}=\left[\begin{array}{lr}
j_{q, k}^{2}-2 q^{k} & -j_{q, k} q^{k} \\
j_{q, k} & -2 q^{k}
\end{array}\right] .
$$

It is easy to see that

$$
\left[\begin{array}{l}
j_{q, k(n+1)} \\
j_{q, k n}
\end{array}\right]=j_{q}\left[\begin{array}{l}
J_{q, k n} \\
J_{q, k(n-1)}
\end{array}\right] \text { and } \Delta\left[\begin{array}{l}
J_{q, k(n+1)} \\
J_{q, k n}
\end{array}\right]=j_{q}\left[\begin{array}{l}
j_{q, k n} \\
j_{q, k(n-1)}
\end{array}\right]
$$

where $J_{q, k n}, j_{q, k n}$ are as above, and $\Delta=1-4 q$.

We obtain Cassini's formula and properties of these numbers by a similar matrix method to the Lucas numbers [KB1].

Proposition 2.2. Let $j_{q}$ be a matrix as in (2.6). Then, for all integers $n \geq 1$, the following matrix power is held below

$$
j_{q}^{n}=\left\{\begin{array}{cr}
\Delta^{\frac{n}{2}}\left[\begin{array}{lr}
J_{q, k(n+1)} & -q^{k} J_{q, k n} \\
J_{q, k n} & -q^{k} J_{q, k(n-1)}
\end{array}\right] \quad \text { if n even } \\
\Delta^{\frac{n-1}{2}}\left[\begin{array}{lr}
j_{q, k(n+1)} & -q^{k} j_{q, k n} \\
j_{q, k n} & -q^{k} j_{q, k(n-1)}
\end{array}\right] \quad \text { if } \mathrm{n} \text { odd },
\end{array}\right.
$$

where $J_{q, k n}$ and $j_{q, k n}$ are the $k n$-th q-Jacobsthal and q-Jacobsthal-Lucas numbers, respectively. 
Proof. We use mathematical induction on $n$. First, we consider odd $n$. For $n=1$,

$$
j_{q}^{1}=\left[\begin{array}{ll}
j_{q, 2 k} & -q^{k} j_{q, k} \\
j_{q, k} & -q^{k} j_{q, 0}
\end{array}\right],
$$

since $j_{q, 2 k}=j_{q, k}^{2}-2 q^{k}$ and $j_{q, 0}=2$. So, (2.7) is indeed true for $n=1$. Now we suppose it is true for $n=t$, that is

$$
j_{q}^{t}=\Delta^{\frac{t-1}{2}}\left[\begin{array}{lr}
j_{q, k(t+1)} & -q^{k} j_{q, k t} \\
j_{q, k t} & -q^{k} j_{q, k(t-1)}
\end{array}\right] .
$$

Using the induction hypothesis and $j_{q}^{2}$ by a direct computation. we can write

$$
j_{q}^{t+2}=j_{q}^{t} j_{q}^{2}=\Delta^{\frac{t+1}{2}}\left[\begin{array}{ll}
j_{q, k(t+3)} & -q^{k} j_{q, k(t+2)} \\
j_{q, k(t+2)} & -q^{k} j_{q, k(t+1)}
\end{array}\right]
$$

as desired. Secondly, let us consider even n. For $n=2$ we can write

$$
j_{q}^{2}=\Delta\left[\begin{array}{cc}
J_{q, 3 k} & -q^{k} J_{q, 2 k} \\
J_{q, 2 k} & -q^{k} J_{q, k}
\end{array}\right] .
$$

So, (2.7) is true for $n=2$. Now, we suppose it is true for $n=t$, using properties of the $q$-Jacobsthal numbers and the induction hypothesis, we can write

$$
j_{q}^{t+2}=\Delta^{\frac{t+2}{2}}\left[\begin{array}{ll}
J_{q, k(t+3)} & -q^{k} J_{q, k(t+2)} \\
J_{q, k(t+2)} & -q^{k} J_{q, k(t+1)}
\end{array}\right],
$$

as desired. Hence, (2.7) holds for all $n$.

If we use the equation (2.5), we can write $\mathrm{j}_{q, k} A_{q}^{n+1}=j_{q, k}^{2} A_{q}^{n}-q^{k} j_{q, k} A_{q}^{n-1}$ $=j_{q, k}^{2} A_{q}^{n}-q^{k}\left(A_{q}^{n}+q^{k} A_{q}^{n-2}\right)$

$=\left(j_{q, k}^{2}-q^{k}\right) A_{q}^{n}-q^{2 k} A_{q}^{n-2}$

Comparing the entries in the first row and first column for the above matrix equation, we get

$$
j_{q, k}=\frac{\left(j_{q, k}^{2}-q^{k}\right) J_{q, k(n+1)}-q^{2 k} J_{q, k(n-1)}}{J_{q, k(n+2)}} .
$$

For $n \geq 0$, if we consider the fact that $\operatorname{det}\left(A_{q}^{n}\right)=\left(\operatorname{det}\left(A_{q}\right)\right)^{n}$, then we obtain the generalized Cassini identity

$$
J_{q, k(n+1)} J_{q, k(n-1)}-J_{q, k n}^{2}=-q^{k(n-1)} J_{q, k}^{2} .
$$


For example, for $k=1$, we get $J_{q, n+1} J_{q, n-1}-J_{q, n}^{2}=-q^{n-1}$, the generalized Cassini identity with $q$-Jacobsthal numbers. In this case, if $q=-1$, we get Cassini identity on classic Fibonacci sequence.

Now we shall derive some results for $\left\{J_{q, k n}\right\}$ by matrix methods.

Proposition 2.3. For all $n, m \in Z$

$$
J_{q, k} J_{q, k(n+m)}=J_{q, k m} J_{q, k(n+1)}-q^{k} J_{q, k(m-1)} J_{q, k n} .
$$

Proof. Since $A_{q}^{n+m}=A_{q}^{n} A_{q}^{m}$ and after some simplifications, we obtain $\mathrm{A}_{q}^{n+m}=\frac{1}{J_{q, k}^{2}}\left[\begin{array}{lr}J_{q, k(n+1)} & -q^{k} J_{q, k n} \\ J_{q, k n} & -q^{k} J_{q, k(n-1)}\end{array}\right]\left[\begin{array}{lr}J_{q, k(m+1)} & -q^{k} J_{q, k m} \\ J_{q, k m} & -q^{k} J_{q, k(m-1)}\end{array}\right]$ $=\frac{J_{q, k m}}{J_{q, k}} A_{q}^{n+1}-q^{k} \frac{J_{q, k(m-1)}}{J_{q, k}} A_{q}^{n}$. Thus we obtain

$$
J_{q, k} A_{q}^{n+m}=J_{q, k m} A_{q}^{n+1}-q^{k} J_{q, k(m-1)} A_{q}^{n},
$$

which, Comparing the entries in the second row and first column for the matrix equation (2.11), gives the conclusion.

When $m=n$ in (2.10), we obtain

$$
J_{q, k} J_{q, 2 k n}=J_{q, k n}\left(J_{q, k(n+1)}-q^{k} J_{q, k(n-1)}\right),
$$

and the follow equality for $J_{q, k n}(x) \neq 0$

$$
J_{q, k} j_{q, k n}=J_{q, k(n+1)}-q^{k} J_{q, k(n-1)} .
$$

Comparing the entries in the first row and first column of the equality (2.11) and by taking $m=n$, we obtain

$$
J_{q, k} J_{q, k(2 n+1)}=J_{q, k(n+1)}^{2}-q^{k} J_{q, k n}^{2},
$$

a particular case of the next equality

$$
J_{q, k} A_{q}^{2 n}=J_{q, k n} A_{q}^{n+1}-q^{k} J_{q, k(n-1)} A_{q}^{n} .
$$

Corollary 2.4. For $k \geq 1$ and $n \in Z$,

$$
\left(2.1 \Phi_{q, k(2 n+1)}+J_{q, k(2 n-1)}=\frac{1}{J_{q, k}}\left(J_{q, k(n+1)}^{2}+\left(1-q^{k}\right) J_{q, k n}^{2}-J_{q, k(n+1)}^{2}\right) .\right.
$$


Proof. Considering the first row and first column of the matrix $A_{q}^{2 n}=$ $\left(A_{q}^{n}\right)^{2}$, we get $J_{q, k} J_{q, k(2 n+1)}=J_{q, k(n+1)}^{2}-q^{k} J_{q, k n}^{2}$, and the second row and second column of the matrix $A_{q}^{2 n}, J_{q, k} J_{q, k(2 n-1)}=J_{q, k n}^{2}-q^{k} J_{q, k(n-1)}^{2}$. By adding side by side in the above equations, we have the conclusion.

For any integer $m$, we have $A_{q}^{2 n}=A_{q}^{n+m} A_{q}^{n-m}$. Here if we consider the entries in the second row and first column in the product $A_{q}^{n+m} A_{q}^{n-m}$ and the matrix $A_{q}^{2 n}$, we get

$$
J_{q, k} J_{q, 2 k n}=J_{q, k(n+m)} J_{q, k(n-m+1)}-q^{k} J_{q, k(n+m-1)} J_{q, k(n-m)} .
$$

\section{3. sums with $q$-Jacobsthal numbers}

We define the $q$-Jacobsthal $G_{q}$-matrix by

$$
G_{q}=\left[\begin{array}{rrr}
1 & 0 & 0 \\
1 & j_{q, k} & -q^{k} \\
0 & 1 & 0
\end{array}\right]
$$

By an inductive argument and using (1.9), we get

Proposition 3.1. For any integer $n \geq 1$ holds:

$$
G_{q}^{n}=\frac{1}{J_{q, k}}\left[\begin{array}{lrr}
1 & 0 & 0 \\
J_{q, k n}^{s} & J_{q, k(n+1)} & -q^{k} J_{q, k n} \\
J_{q, k(n-1)}^{s} & J_{q, k n} & -q^{k} J_{q, k(n-1)}
\end{array}\right],
$$

where $J_{q, k n}^{s}$ is defined such that $J_{q, k n}^{s}=\sum_{i=1}^{n} J_{q, k i}$.

Proof. (By induction). For $n=1$ :

$$
G_{q}^{1}=\left[\begin{array}{rrr}
1 & 0 & 0 \\
1 & j_{q, k} & -q^{k} \\
0 & 1 & 0
\end{array}\right]=\frac{1}{J_{q, k}}\left[\begin{array}{rrr}
1 & 0 & 0 \\
J_{q, k}^{s} & J_{q, 2 k} & -q^{k} J_{q, k} \\
J_{q, 0}^{s} & J_{q, k} & -q^{k} J_{q, 0}
\end{array}\right]
$$

since $J_{q, 0}=0$ and $J_{q, 2 k}=j_{q, k} J_{q, k}$. Let us suppose that the formula is true for $n-1$ :

$$
G_{q}^{n-1}=\frac{1}{J_{q, k}}\left[\begin{array}{lrr}
1 & 0 & 0 \\
J_{q, k(n-1)}^{s} & J_{q, k n} & -q^{k} J_{q, k(n-1)} \\
J_{q, k(n-2)}^{s} & J_{q, k(n-1)} & -q^{k} J_{q, k(n-2)}
\end{array}\right]
$$


Then,

$$
\begin{aligned}
& \mathrm{G}_{q}^{n}=G_{q}^{n-1} G_{q}^{1}=\frac{1}{J_{q, k}}\left[\begin{array}{lrr}
1 & 0 & 0 \\
J_{q, k(n-1)}^{s} & J_{q, k n} & -q^{k} J_{q, k(n-1)} \\
J_{q, k(n-2)}^{s} & J_{q, k(n-1)} & -q^{k} J_{q, k(n-2)}
\end{array}\right]\left[\begin{array}{rrr}
1 & 0 & 0 \\
1 & j_{q, k} & -q^{k} \\
0 & 1 & 0
\end{array}\right] \\
& =\frac{1}{J_{q, k}}\left[\begin{array}{lrr}
1 & 0 & 0 \\
J_{q, k(n-1)}^{s}+J_{q, k n} & j_{q, k} J_{q, k n}-q^{k} J_{q, k(n-1)} & -q^{k} J_{q, k n} \\
J_{q, k(n-2)}^{s}+J_{q, k(n-1)} & j_{q, k} J_{q, k(n-1)}-q^{k} J_{q, k(n-2)} & -q^{k} J_{q, k(n-1)}
\end{array}\right] \\
& =\frac{1}{J_{q, k}}\left[\begin{array}{lrr}
1 & 0 & 0 \\
J_{q, k n}^{s} & J_{q, k(n+1)} & -q^{k} J_{q, k n} \\
J_{q, k(n-1)}^{s} & J_{q, k n} & -q^{k} J_{q, k(n-1)}
\end{array}\right] .
\end{aligned}
$$

Corollary 3.2. If $J_{q, k n}$ is the $k n$-th $q$-Jacobsthal number, then

$$
J_{q, k} J_{q, k(n+m)}^{s}=J_{q, k n}^{s}+J_{q, k(m-1)}^{s}\left(J_{q, k(n+1)}-q^{k} J_{q, k n}\right)+J_{q, k(n+1)} J_{q, k m} .
$$

Proof. The second row and first column in the matrix $G_{q}^{n+m}$ is equal to $J_{q, k(n+m)}^{s}$. Considering the $(2,1)$-entries of the matrix equation $G_{q}^{n} G_{q}^{m}$,

$\mathrm{J}_{q, k} J_{q, k(n+m)}^{s}=J_{q, k n}^{s}+J_{q, k(n+1)} J_{q, k m}^{s}-q^{k} J_{q, k n} J_{q, k(m-1)}^{s}$

$=J_{q, k n}^{s}+J_{q, k(n+1)}\left(J_{q, k(m-1)}^{s}+J_{q, k m}\right)-q^{k} J_{q, k n} J_{q, k(m-1)}^{s}$

$=J_{q, k n}^{s}+J_{q, k(m-1)}^{s}\left(J_{q, k(n+1)}-q^{k} J_{q, k n}\right)+J_{q, k(n+1)} J_{q, k m}$. Thus, the proof is completed.

If $q=-2$ and $k=1$, the classic Jacobsthal sequence satisfy

$$
J_{n+m}^{s}=J_{n}^{s}+J_{m-1}^{s} J_{n+2}+J_{n+1} J_{q, m} .
$$

\section{4. conclusion}

In this paper, we consider the amazing relationships between the $q$-Jacobsthal numbers and matrices. Sum formula involving the terms of $q$-Jacobsthal numbers is one of the most important results obtained in this study.

Note that the $q$-Jacobsthal numbers represent a generalization of the classical Fibonacci sequence. In particular, we have obtained new results by matrix method in these numbers. 


\section{References}

[C1] G. Cerda-Morales, On generalized Fibonacci and Lucas numbers by matrix methods, Hacettepe J. Math. Stat. (to appear).

[C2] G. Cerda-Morales, Matrix methods in Horadam Sequences, Boletín de matemáticas, Universidad Nacional de Colombia, Vol. 19, no. 1, pp. 55-64, (2012).

[H1] A.F. Horadam, Basic properties of a certain generalized sequence of numbers, Fibonacci Quarterly, Vol. 3, pp. 161-176, (1965).

[H2] A. F. Horadam, Jacobsthal Representation Numbers, The Fibonacci Quarterly, Vol. 34, no. 1, pp. 40-53, (1996).

[KB1] F. Köken, D. Bozkurt, On Lucas numbers by the matrix method, Hacettepe Journal of Mathematics and Statistics, Vol. 39, no. 4, pp. 471-475, (2010).

[KB2] F. Köken, D. Bozkurt, On The Jacobsthal numbers by matrix methods, Int. J. Contemp. Math. Sciences, Vol. 3, no. 13, pp. 605-614, (2008).

[KB3] F. Köken, D. Bozkurt, On The Jacobsthal-Lucas Numbers by Matrix Methods, Int. J. Contemp. Math. Sciences, Vol. 3, no. 33, pp. 1629-1633, (2008).

[KM] D. Kalman, R. Mena, The Fibonacci numbers- exposed, Math. Mag., no. 76 , pp. 81-167, 2003.

[KS] E. Kilic, P. Stanica, Factorizations and representations of second linear recurrences with indices in arithmetic progressions, Bol. Mex. Math. Soc., Vol. 15, no. 1, pp. 23-36, (2009).

[Ko] T. Koshy, Fibonacci and Lucas Numbers with Applications, A WileyInterscience Publications, New York, (2002). 


\section{Gamaliel Cerda-Morales}

Instituto de Matemáticas

P. Universidad Católica de Valparaíso

Blanco Viel 596

Cerro Barón

Valparaíso

Chile

e-mail : gamaliel.cerda.m@mail.pucv.cl 